\title{
IUFOST2006/1016 Harmonisation and validation of methods in Food Safety
}

\author{
N. Cook \\ DEFRA Central Science Laboratory, Sand Hutton, YO1 1LZ York, United Kingdom \\ n.cook@csl.gov.uk
}

In order that analytical methods conform to basic common principles, it is necessary to harmonise the steps which are undertaken in their development, and also harmonise the criteria for their performance and interpretation of their results. Such harmonisation fundamentally requires a high level of communication between methods developers, predominantly at an international level. The fragmentation which exists between European scientists active in methods development for Food Safety and Quality analysis has been a major barrier to effective harmonisation of the analytical methods which have been and are being produced through their activities. Proper validation based on consensus criteria is an absolute prerequisite for successful implementation. Most current standards were initially prepared without available validation data, which has had to be provided later with some considerable effort. The development of standardised methods requires the activity of expert laboratories, along with the collaboration of end-user laboratories for validation of these methods. Validation activities require major international efforts and extensive organisation to set up and conduct. To date the various European methods validation projects have been conducted somewhat insularly. This has been exacerbated by a lack of strong contact with the end-user and stakeholder. Implementation of methods in food safety analysis will benefit from a concerted approach to harmonisation and validation, with involvement and cooperation of the various stakeholders concerned. 